\title{
Multipath Simulator Measurements of Handset Dual Antenna Performance With Limited Number of Signal Paths
}

\author{
Paul Hallbjörner, Juan D. Sánchez-Heredia, Peter Lindberg, Member, IEEE, Antonio M. Martínez-González, and
} Thomas Bolin

\begin{abstract}
Antenna pairs for diversity or MIMO functionality are characterized under the assumption of a certain statistical distribution of the incident signals over angle and polarization, but also assuming a signal environment with a large number of signal paths. In many real-life environments, however, only a few signal paths contain most of the transferred power. A multipath simulator can be used to realize signal environments with a controlled number of signal paths. This paper presents measurements of dual antenna performance using a multipath simulator with 2-16 signal paths. The results are analyzed in terms of statistical power distributions, power imbalance, correlation coefficient, multiplexing efficiency, and diversity gain. Differences in performance depending on the number of signal paths are noted, illustrating the value of considering the number of signal paths in characterization.
\end{abstract}

Index Terms-Antenna measurements, multipath simulator, signal environment, sparse environment, terminal antenna.

\section{INTRODUCTION}

D UAL antennas in mobile terminals are used more and more, for diversity or multiple-input multiple-output (MIMO) functionality. There are different opinions regarding the proper way to characterize antenna pairs for diversity or MIMO functionality, involving different parameters [1]. One common way to characterize dual antennas is by their mean effective gains (MEG) and correlation coefficient [2], [3]. These parameters depend on the assumed statistical distribution of the incident signals over angle and polarization. Dual antenna performance is also based on the assumption of a large number of signal paths [4], [5]. When calculating the correlation coefficient from the radiation patterns of the two antennas, the integrals are in effect summations of an infinite number of signal paths. There is, however, nothing in the integral about the number of simultaneous signal paths at any instant.

Manuscript received April 20, 2011; revised September 20, 2011; accepted October 10, 2011. Date of publication October 25, 2011; date of current version February 03, 2012. The work was supported in part by MICINN (Project TEC2008-05811) through an FPI doctoral grant (BES-2009-013764).

P. Hallbjörner is with SP Technical Research Institute of Sweden, SE-501 15 Borås, Sweden (e-mail: paul.hallbjorner@sp.se).

J. D. Sánchez-Heredia and A. M. Martínez-González are with the Universidad Politécnica de Cartagena (UPCT), E-30202 Cartagena, Spain (e-mail: jd.sanchez@upct.es; toni.martinez@upct.es).

P. Lindberg is with TE Connectivity, SE-175 26 Järfälla, Sweden (e-mail: peter.lindberg@te.com).

T. Bolin is with Sony Ericsson Mobile Communications, Nya Vattentornet, SE-221 88 Lund, Sweden (e-mail: thomas.bolin@sonyericsson.com).

Digital Object Identifier 10.1109/TAP.2011.2173451

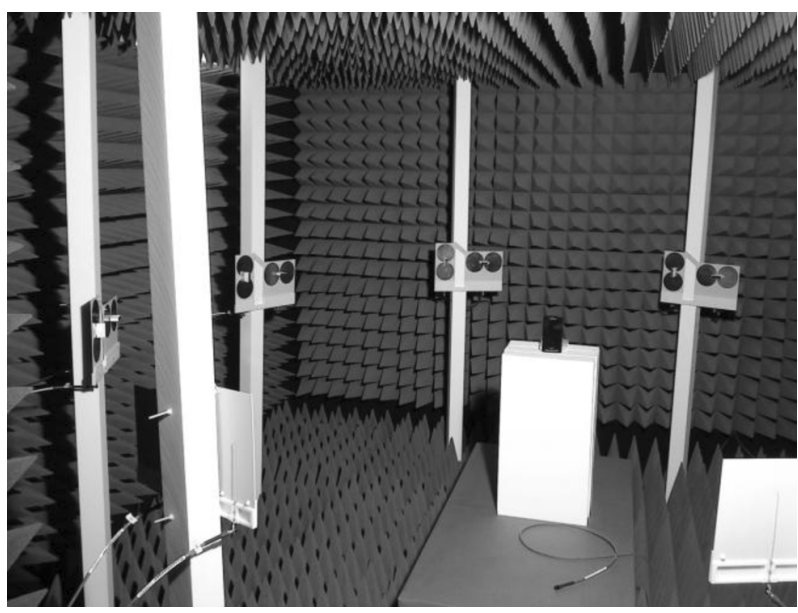

Fig. 1. MPS in anechoic room, with test object at the center of the MPS array.

In many real-life environments, most of the transferred power is contained in only a few signal paths [6], [7]. The performance of an antenna pair in such environments can be studied by computer simulations using the radiation patterns. Dual antenna performance in multipath environments can also be measured directly, without going via the radiation patterns [8]. Reverberation chambers (RC) [9], [10] is an established technique for this. RCs are usually designed so as to ensure that the test object is subjected to a large number of signal paths. Multipath simulators (MPS) [11]-[13] are an emerging technique that allows better control of the signal environment than RCs. For instance, the number of signal paths is a parameter that can be decided arbitrarily.

This paper presents a study in which a multipath simulator is used to characterize dual antenna performance in environments with a limited number of signal paths. The purpose is to have experimental indications of how performance typically differs depending on the number of signal paths, and thereby also indications regarding the need to consider the number of signal paths in characterization.

\section{SETUP}

\section{A. Multipath Simulator}

An MPS comprising 16 antennas is used. The antennas are of vertical and horizontal polarizations, and evenly distributed on a circle around the test object, in a plane, see Fig. 1. 


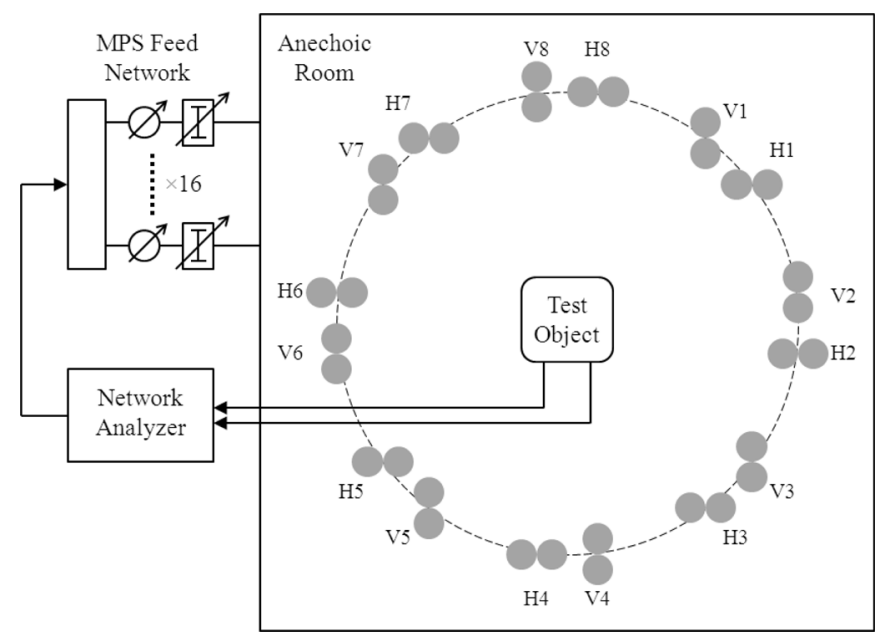

Fig. 2. Block diagram of the setup for S-parameter measurements on dual antennas.

TABLE I

Phase SweEP Rate ON the MPS BRANCHES, AND the ACtive BRANCHES USED For the Different Number of Paths. For 2, 4, AND 8 PATHS, Three Sets ARe Used, EACH Given by a COLUMn

\begin{tabular}{|c|c|c|c|c|c|c|c|c|c|c|c|}
\hline $\begin{array}{c}\text { MPS } \\
\text { Branch }\end{array}$ & $\begin{array}{c}\text { Phase Sweep } \\
\text { Rate }(\lambda / s)\end{array}$ & \multicolumn{3}{|c|}{$\begin{array}{c}2 \\
\text { Paths }\end{array}$} & \multicolumn{3}{|c|}{$\begin{array}{c}4 \\
\text { Paths }\end{array}$} & \multicolumn{3}{|c|}{$\begin{array}{c}8 \\
\text { Paths }\end{array}$} & $\begin{array}{c}16 \\
\text { Paths }\end{array}$ \\
\hline V1 & 40.16 & $\mathrm{O}$ & $\mathrm{O}$ & - & $\mathrm{O}$ & $\mathrm{O}$ & - & $\mathrm{O}$ & $\mathrm{O}$ & - & $\mathrm{O}$ \\
\hline H1 & 45.20 & - & - & - & - & - & - & - & $\mathrm{O}$ & - & $\mathrm{O}$ \\
\hline V2 & 2.45 & - & - & $\mathrm{O}$ & $\mathrm{O}$ & - & $\mathrm{O}$ & $\mathrm{O}$ & - & $\mathrm{O}$ & $\mathrm{O}$ \\
\hline $\mathrm{H} 2$ & 12.15 & - & - & - & - & - & - & - & - & $\mathrm{O}$ & $\mathrm{O}$ \\
\hline V3 & 7.34 & - & - & - & - & - & - & - & $\mathrm{O}$ & - & $\mathrm{O}$ \\
\hline $\mathrm{H} 3$ & 16.84 & - & - & - & - & $\mathrm{O}$ & - & $\mathrm{O}$ & $\mathrm{O}$ & - & $\mathrm{O}$ \\
\hline V4 & 47.08 & - & - & - & - & - & - & - & - & $\mathrm{O}$ & $\mathrm{O}$ \\
\hline $\mathrm{H} 4$ & 49.46 & $\mathrm{O}$ & - & - & - & - & $\mathrm{O}$ & $\mathrm{O}$ & - & $\mathrm{O}$ & $\mathrm{O}$ \\
\hline V5 & 48.50 & - & - & - & - & $\mathrm{O}$ & - & $\mathrm{O}$ & $\mathrm{O}$ & - & $\mathrm{O}$ \\
\hline H5 & 49.94 & - & $\mathrm{O}$ & - & $\mathrm{O}$ & - & - & - & $\mathrm{O}$ & - & $\mathrm{O}$ \\
\hline V6 & 37.05 & - & - & - & - & - & $\mathrm{O}$ & $\mathrm{O}$ & - & $\mathrm{O}$ & $\mathrm{O}$ \\
\hline H6 & 42.89 & - & - & $\mathrm{O}$ & $\mathrm{O}$ & - & - & - & - & $\mathrm{O}$ & $\mathrm{O}$ \\
\hline V7 & 25.71 & - & - & - & - & - & - & - & $\mathrm{O}$ & - & $\mathrm{O}$ \\
\hline H7 & 33.58 & - & - & - & - & $\mathrm{O}$ & - & $\mathrm{O}$ & $\mathrm{O}$ & - & $\mathrm{O}$ \\
\hline V8 & 21.38 & - & - & - & - & - & - & - & - & $\mathrm{O}$ & $\mathrm{O}$ \\
\hline $\mathrm{H} 8$ & 29.78 & - & - & - & - & - & $\mathrm{O}$ & $\mathrm{O}$ & - & $\mathrm{O}$ & $\mathrm{O}$ \\
\hline
\end{tabular}

The MPS thus simulates a 2-D signal environment with up to 16 signal paths. Each MPS antenna is fed via a sweeping phase shifter, which simulates the Doppler shift experienced when the terminal moves in a multipath environment. By setting the sweep rate differently on the antennas, a fast fading signal is received by the test object antennas. Each MPS antenna is also fed via an attenuator, and the antenna is effectively switched on/off by setting its attenuator to minimum/maximum attenuation.

Fig. 2 shows a block diagram of the setup, with a network analyzer for simultaneous measurements of the complex transmission coefficients $\left(S_{21}\right)$ between the MPS port and each of the two antenna ports of the test object.

Each measurement in the presented work is a $20 \mathrm{~s}$ sequence of $2 \times 6401 S_{21}$ values between the MPS port and the two test object antenna connectors. A maximum Doppler shift of $50 \mathrm{~Hz}$ is used, to ensure that samples are taken with sufficient density with respect to the fast fading. Table I shows the phase sweep rates over the MPS antenna array that realize the desired Doppler shifts. Measurements are performed with 2, 4, 8, and 16 MPS antennas active, and the antennas that are switched on all have equal amplitude. Which MPS antennas are active for the respective number of signal paths is also shown in Table I, with reference to Fig. 2 for the physical locations of the antennas. Active antennas are marked in Table I with an "O". With 2, 4, and 8 paths, there are three different sets of active antennas, given by the three columns.

\section{B. Performance Metrics}

Results are analyzed in terms of the power imbalance $\delta$, correlation coefficient $\rho_{c}$, and multiplexing efficiency $\eta_{\operatorname{mux}}$ [14], according to the expressions

$$
\begin{aligned}
\delta & =\frac{\left\langle\left|S_{21,1}\right|^{2}\right\rangle}{\left\langle\left|S_{21,2}\right|^{2}\right\rangle} \\
\rho_{c} & =\frac{\left\langle\left(S_{21,1}-\left\langle S_{21,1}\right\rangle\right)\left(S_{21,2}-\left\langle S_{21,2}\right\rangle\right)^{*}\right\rangle}{\sqrt{\left\langle\left|S_{21,1}-\left\langle S_{21,1}\right\rangle\right|^{2}\right\rangle\langle| S_{21,2}-\left\langle\left. S_{21,2}\right|^{2}\right\rangle}} \\
\eta_{\text {mux }} & =2 \frac{\sqrt{\left\langle\left|S_{21,1}\right|^{2}\right\rangle\left\langle\left|S_{21,2}\right|^{2}\right\rangle\left(1-\left|\rho_{c}\right|^{2}\right)}}{\left\langle\left|S_{21,1}\right|^{2}\right\rangle+\left\langle\left|S_{21,2}\right|^{2}\right\rangle} .
\end{aligned}
$$

In (1)-(3), $S_{21,1}$ and $S_{21,2}$ are the complex transmission coefficients of the respective test object antenna, each being a sequence of 6401 samples, and $\langle\cdot\rangle$ denotes average over the whole sequence. The expression in (3) differs from the one in [14] in that normalization to the average radiation efficiency of the two antennas is introduced. This is done because we want to study only the effects of imbalance and correlation, but not the effect of the absolute levels of the efficiencies.

Diversity gain $G_{d}$ [15] is also used as a figure-of-merit. Maximum ratio combining is assumed, and the combined signal is thus

$$
S_{21, c}=\sqrt{\left|S_{21,1}\right|^{2}+\left|S_{21,2}\right|^{2}} .
$$

After this, $G_{d}$ is calculated at the $99 \%$ signal reliability levels,

$$
G_{d, k}=\frac{\left|S_{21, c, 99 \%}\right|^{2}}{\left|S_{21, k, 99 \%}\right|^{2}}
$$

where two values are calculated ( $k=1$ and $k=2$ ) with each of the two antennas serving as the non-diversity reference case. The reason for doing this, rather than using one specific antenna as the reference, is that we hereby avoid assuming which antenna would be used in a non-diversity case.

\section{MEASUREMENTS}

Two experiments are carried out, with different test objects. In the first, a test object with near-ideal performance in an isotropic environment is used. This makes an interesting reference case for the change in performance when going to fewer signal paths. In the second experiment, two terminal prototypes with less ideal and more typical performance are used. They are prototypes of the same terminal, but with different design solutions for the dual antennas. This represents a realistic situation in which different designs are characterized and compared. 


\section{A. Near-Ideal Test Object}

A terminal antenna model for the $2600 \mathrm{MHz}$ band is used, with measurements performed at $2655 \mathrm{MHz}$. The model is made of metal only (no plastic housing) and consists of a $100 \mathrm{~mm} \times 40 \mathrm{~mm}$ ground plane with two PIFA antennas fed by coaxial cables. The cables are routed so as to minimize influence on the antenna performance [16]. Tests with different feed cable arrangements and with ferrite absorbers [17] are performed to ensure that the results are not affected too much. Electrical performance is a return loss of $12 \mathrm{~dB}$ and near $100 \%$ radiation efficiency on both antennas, and a correlation coefficient of 0.25 in an isotropic environment with a large number of signal paths.

In the MPS measurements, the test object is mounted in freespace (no head or hand) at the center of the MPS array. It is measured in three orthogonal planes with each set of active branches according to Table I. It is also measured in a $45^{\circ}$ slant orientation (data mode), with 10 different orientations in azimuth. The slant orientation measurements are done only for the leftmost set of active branches for 2 and 4 paths, and the set of 16 branches. The total number of measurements are thus $19 / 19 / 9 / 13$ for the respective $2 / 4 / 8 / 16$ paths.

Fig. 3 shows its performance for the different number of signal paths. Power imbalance, correlation coefficient, and multiplexing efficiency all show a strong increase in the spread when reducing the number of signal paths. The performance with 16 signal paths agrees well with the performance in an isotropic environment with a large number of signal paths, with only small variations. With two signal paths, the correlation coefficient is many times very high, and even with four signal paths it varies over almost all possible values. With few signal paths, the average correlation coefficient is also increased significantly compared to the case of many signal paths.

Examples of time sequences and cumulative distribution functions (CDF) of the received power are plotted for a few measurements. The case of two signal paths are shown in Fig. 4(a) and (b), while the case of 16 signal paths are seen in Fig. 4(c) and (d). With 16 paths, time sequences are similar to Rayleigh fading curves, and the CDF curves are all approximately the same, corresponding to the theoretical curves under assumption of a large number of signal paths. With two paths, on the other hand, strong variations are seen in time sequence characteristics and CDF curve shapes. As a result, $G_{d}$ can be anything from very large to almost none.

\section{B. Two Realistic Test Objects}

Two samples of the same terminal model, with dimensions $115 \mathrm{~mm} \times 65 \mathrm{~mm}$, but with different antenna solutions, are measured. The two test objects are referred to as Prototype A and Prototype B. These models are more realistic than the first one, having plastic housing and antennas with less ideal performance. Their antennas are made for the $700 \mathrm{MHz}$ band, and the measurements are performed at $740 \mathrm{MHz}$. The antennas are accessed via coaxial cables, which exit the test objects on the middle of the long side for minimum interference. As with the near-ideal test object, tests are conducted to ensure that the feed cables have a negligible effect on performance. The respective antenna performance in a 3-D environment with a large number

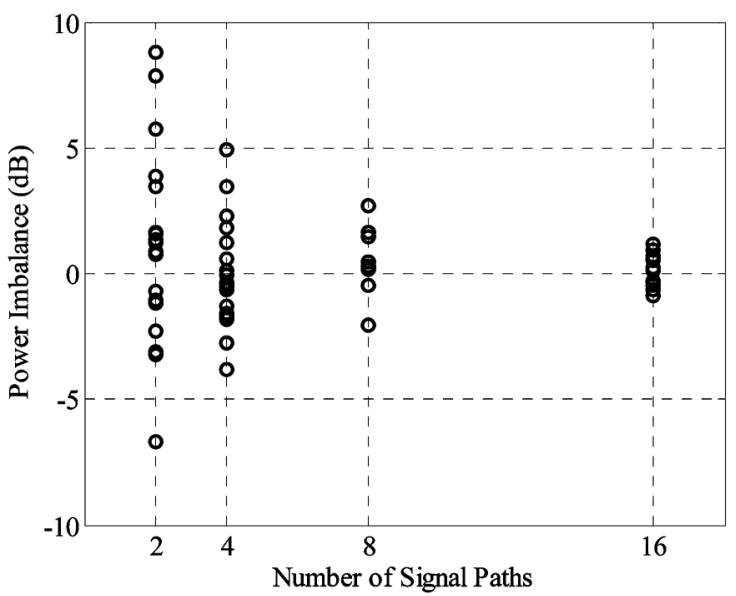

(a)

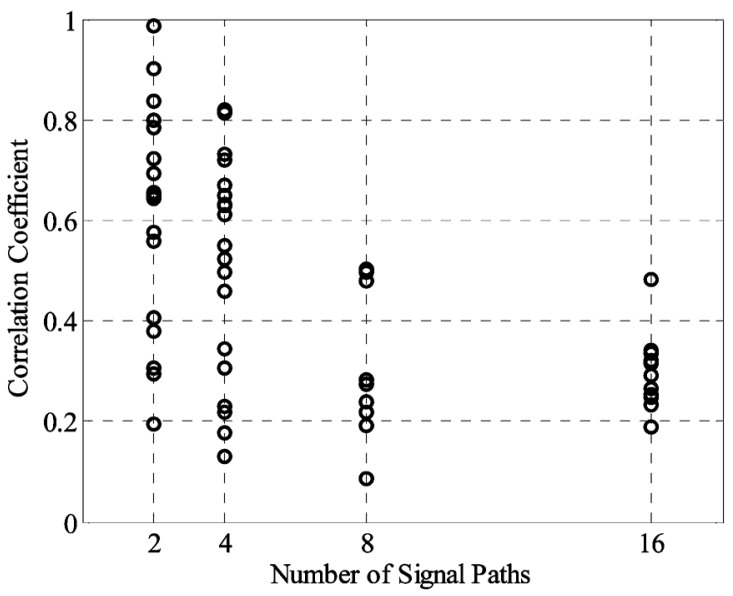

(b)

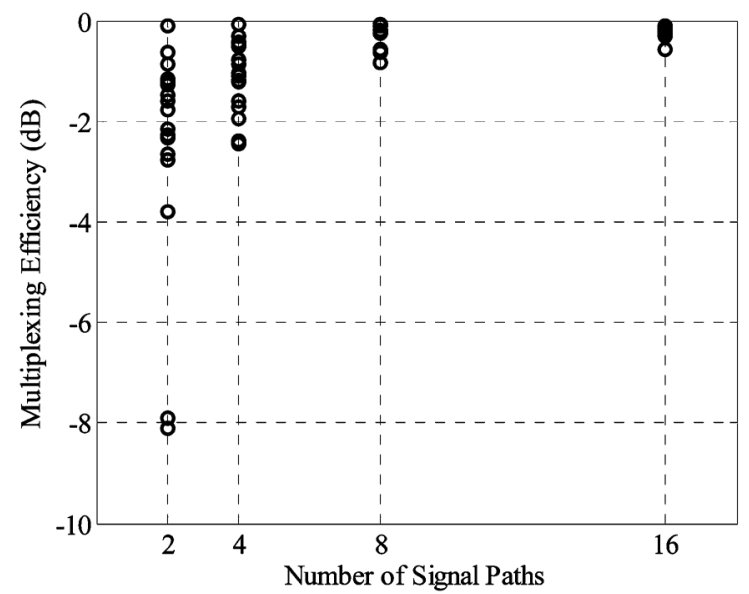

(c)

Fig. 3. (a) Power imbalance $\delta$ of the near-ideal test object, as a function of the number of signal paths. (b) Correlation coefficient $\left|\rho_{c}\right|$ of the near-ideal test object, as a function of the number of signal paths. (c) Multiplexing efficiency $\eta_{\text {mux }}$ of the near-ideal test object, as a function of the number of signal paths.

of signal paths is measured in an RC. The power imbalances are $-0.8 \mathrm{~dB}$ for Prototype $\mathrm{A}$ and $-0.3 \mathrm{~dB}$ for Prototype $\mathrm{B}$, and the correlation coefficients are 0.35 for Prototype A and 0.56 for Prototype B. More interesting for comparison with the MPS measurements is the performance in a 2-D environment. These values are calculated from 2 -D cuts of the radiation patterns in 

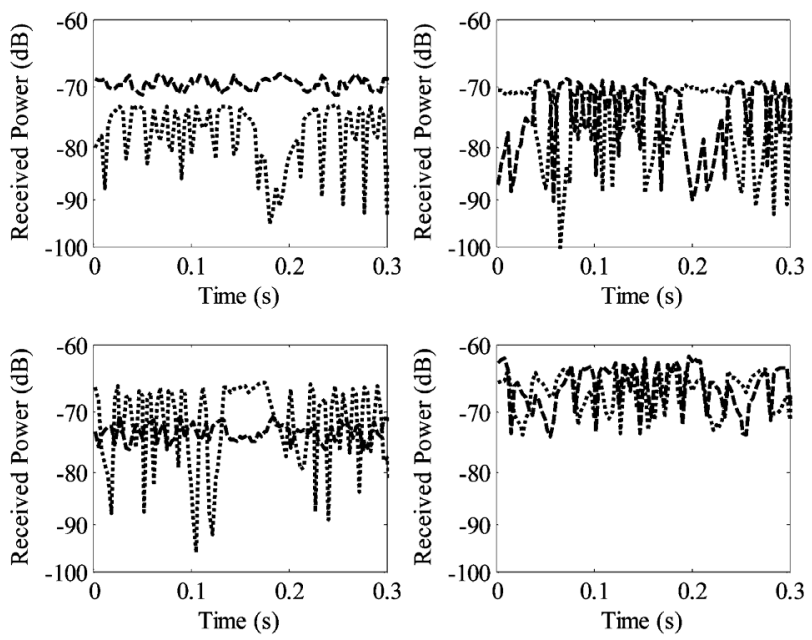

(a)
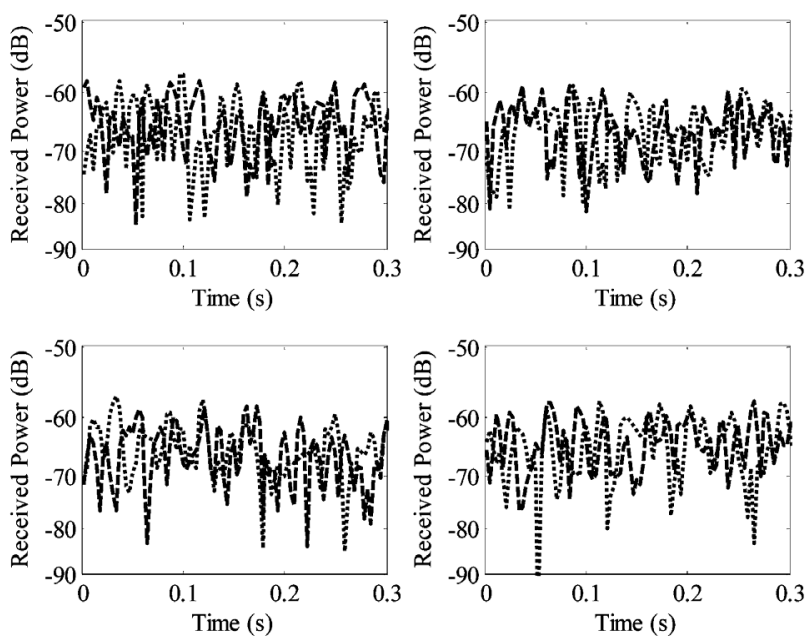

(c)
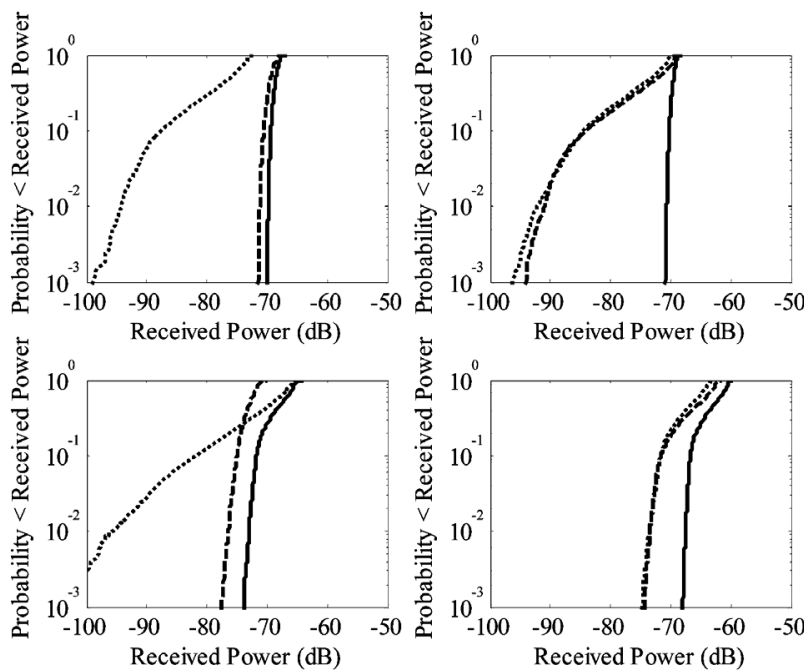

(b)
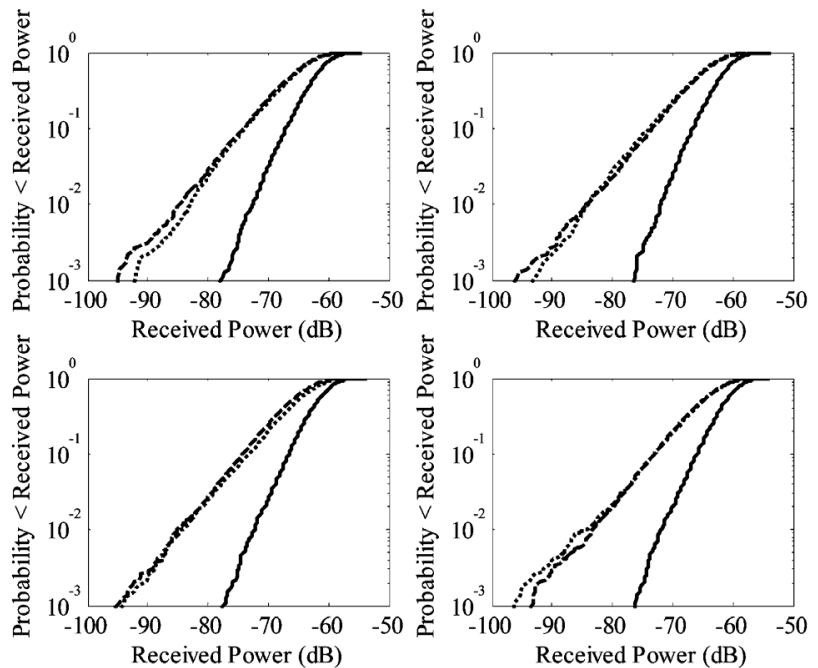

(d)

Fig. 4. (a) Examples of time sequences for the case of two signal paths, on the two branches (dotted and dashed). (b) Examples of cumulative distribution functions for the case of two signal paths, with the two branches (dotted and dashed) and the combined signal using maximum ratio combining (solid). The four examples show vastly different diversity gains. (c) Examples of time sequences for the case of 16 signal paths, on the two branches (dotted and dashed). (d) Examples of cumulative distribution functions for the case of 16 signal paths, with the two branches (dotted and dashed) and the combined signal using maximum ratio combining (solid). All four examples are approximately the same.

TABLE II

Power IMbalance AND CORRELATION COEFFICIENT OF PRototypes A AND B, IN DIFFERENT 2-D ENVIRONMENTS

\begin{tabular}{|c|l|c|c|c|}
\hline \hline Prot. & Parameter & XY-Plane & XZ-Plane & YZ-Plane \\
\hline A & Power imbalance (dB) & -0.4 & 1.5 & 0.7 \\
B & Power imbalance (dB) & -1.0 & -0.3 & 0.0 \\
A & Correlation coefficient & 0.60 & 0.75 & 0.70 \\
B & Correlation coefficient & 0.68 & 0.81 & 0.56 \\
\hline
\end{tabular}

each of three orthogonal planes. The cuts are measured with a standard far-field antenna test range. Uniform power distribution over angle and polarization is assumed when calculating the performance parameter from these 2-D cuts. As can be seen in Table II, the test objects are well balanced in all three planes. Correlation coefficients are notably higher in the 2-D environments than in the 3-D environment.

For the MPS measurements, the test objects are mounted in free-space at the center of the MPS array. They are measured in three orthogonal planes with each set of active branches according to Table I. The same planes and orientations are used for both test objects. The total number of measurements for each test object are thus 9/9/9/3 for the respective 2/4/8/16 paths.

Results are seen in Fig. 5, with circles for Prototype A and triangles for Prototype B. The plots show that also in this experiment, reducing the number of signal paths leads to a much greater spread in the different metrics. Power imbalance is not as symmetrical around $0 \mathrm{~dB}$ as in the first experiment, and Prototype A shows a larger spread than Prototype B. For both test objects, $\left|\rho_{c}\right|$ is clearly worse with 16 paths than in a 3-D environment with many paths, but comparable to 2-D environments according to Table II. At 16 signal paths, $\left|\rho_{c}\right|$ is higher for Prototype B than Prototype A, and it is significantly higher with fewer signal paths. A correlation coefficient of 0.7 is usually considered the limit for good diversity/MIMO performance. Table III shows the proportion of $\left|\rho_{c}\right|$ values less than 0.7 for the two prototypes, indicating that Prototype A is superior. The spread 


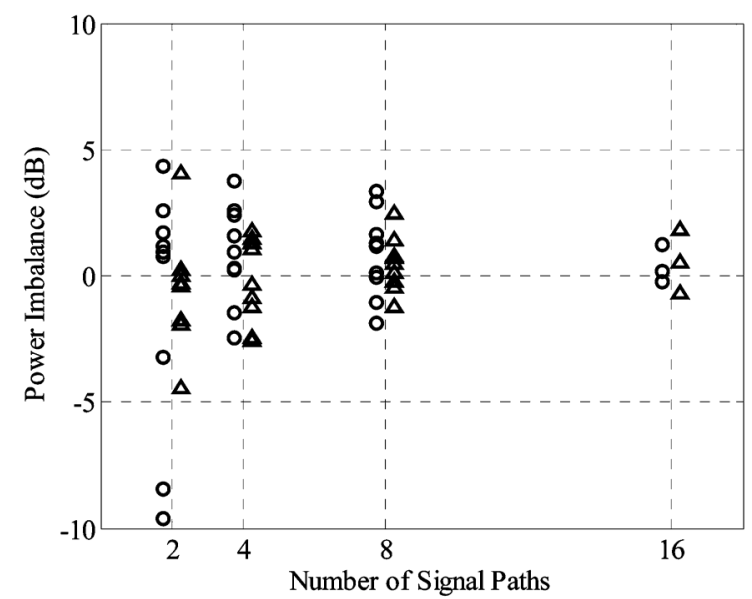

(a)

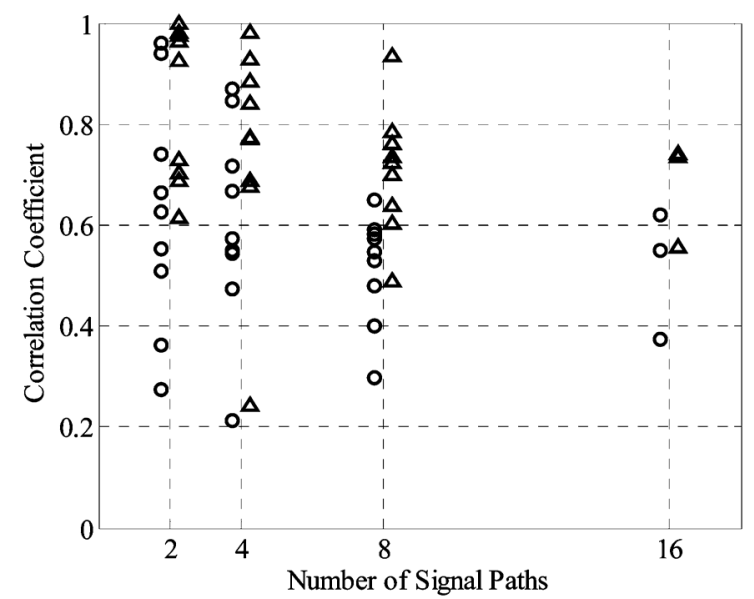

(b)

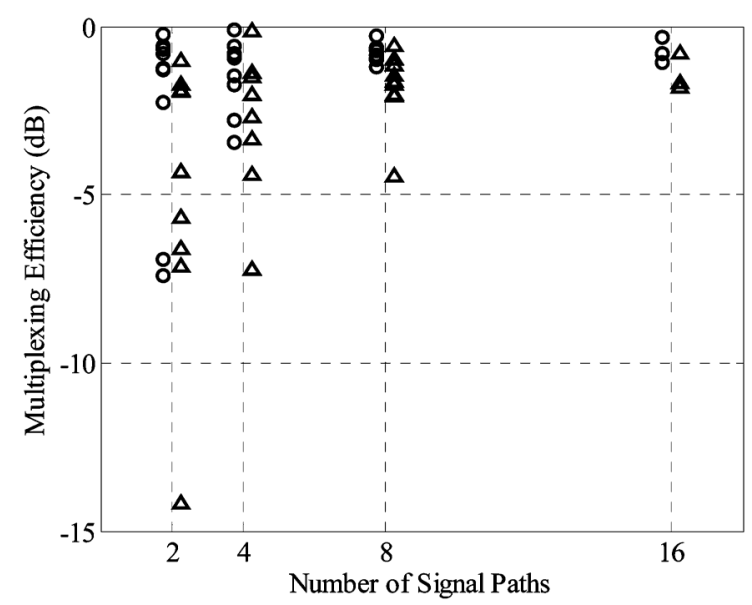

(c)

Fig. 5. (a) Power imbalance $\delta$ of Prototype A (circles) and Prototype B (triangles), as a function of the number of signal paths. (b) Correlation coefficient $\left|\rho_{c}\right|$ of Prototype A (circles) and Prototype B (triangles), as a function of the number of signal paths. (c) Multiplexing efficiency $\eta_{m u x}$ of Prototype A (circles) and Prototype B (triangles), as a function of the number of signal paths.

is however very large for both models, and there are many cases in which the performance difference is reversed.

The multiplexing efficiency, being a function of both the efficiencies and the correlation, show better performance for Prototype A in general, and a quicker degradation for Prototype B
TABLE III

PROPORTION OF CORRELATION COEFFICIENT VALUES LESS THAN 0.7 FOR PRototypes A AND B, FOR DifFERENT Number of Signal Paths

\begin{tabular}{|c|l|c|c|c|c|}
\hline \hline Prot. & Parameter & 2 Paths & 4 Paths & 8 Paths & 16 Paths \\
\hline A & Proportion of $\rho_{c} \mid<0.7$ & $67 \%$ & $67 \%$ & $100 \%$ & $100 \%$ \\
B & Proportion of $\rho_{c} \mid<0.7$ & $22 \%$ & $33 \%$ & $44 \%$ & $33 \%$ \\
\hline \hline
\end{tabular}

TABLE IV

Median and Worst Case Multiplexing EfFiciency of Prototypes A AND B, FOR DifFERENT NUMBER OF Signal PATHS

\begin{tabular}{|c|l|c|c|c|c|}
\hline \hline Prot. & Parameter & 2 Paths & 4 Paths & 8 Paths & 16 Paths \\
\hline A & Median $\eta_{m u x}(\mathrm{~dB})$ & -1.3 & -0.9 & -0.9 & -0.8 \\
B & Median $\eta_{m u x}(\mathrm{~dB})$ & -4.3 & -2.0 & -1.6 & -1.7 \\
A & Worst case $\eta_{\operatorname{mux}}(\mathrm{dB})$ & -7.4 & -3.5 & -1.2 & -1.1 \\
B & Worst case $\eta_{m u x}(\mathrm{~dB})$ & -14.2 & -7.2 & -4.5 & -1.8 \\
\hline \hline
\end{tabular}

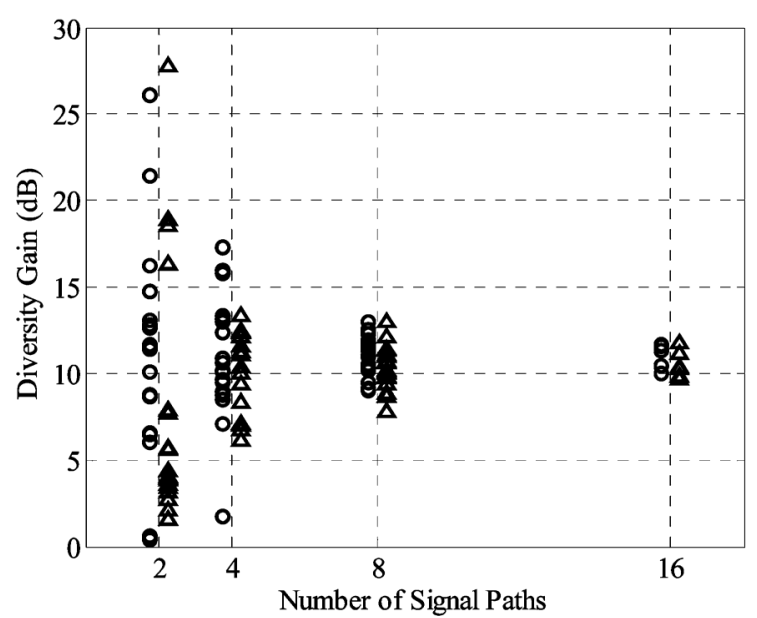

Fig. 6. Diversity gain $G_{d}$ of Prototype A (circles) and Prototype B (triangles), using maximum ratio combining, as a function of the number of signal paths.

TABLE V

Best Case, Median, and Worst Case Diversity Gain of Prototypes A AND B, FOR DiFFERENT NUMBER OF SIGNAL PATHS

\begin{tabular}{|c|l|c|c|c|c|}
\hline \hline Prot. & Parameter & 2 Paths & 4 Paths & 8 Paths & 16 Paths \\
\hline A & Best case $G_{d}(\mathrm{~dB})$ & 26.1 & 17.3 & 13.0 & 11.6 \\
B & Best case $G_{d}(\mathrm{~dB})$ & 27.8 & 13.3 & 12.9 & 11.7 \\
A & Median $G_{d}(\mathrm{~dB})$ & 11.5 & 10.4 & 11.1 & 11.4 \\
B & Median $G_{d}(\mathrm{~dB})$ & 4.2 & 10.7 & 10.4 & 10.3 \\
$\mathrm{~A}$ & Worst case $G_{d}(\mathrm{~dB})$ & 0.4 & 1.7 & 9.0 & 10.0 \\
B & Worst case $G_{d}(\mathrm{~dB})$ & 1.6 & 6.1 & 7.8 & 9.6 \\
\hline \hline
\end{tabular}

as the number of signal paths is reduced. Table IV summarizes the median and worst case multiplexing efficiencies.

Diversity gain is shown in Fig. 6 and Table V. From 16 paths down to four, the median $G_{d}$ remains stable for both prototypes, but with an increase in the spread. With two paths, the median value for Prototype B drops drastically, while it remains approximately the same for Prototype A. The spread increases even more for both, and Prototype B has better best case and worst case values. Best and worst cases are however highly sensitive to random fluctuations. Which model has the best diversity gain performance depends on which number of signal paths and which statistical parameter (median, best/worst case) is considered. 


\section{CONCLUSION}

Mobile terminal dual antennas are characterized using an MPS, which provides a 2-D signal environment with up to 16 signal paths. Three test objects are characterized with $2,4,8$, and 16 simulated signal paths.

Several conclusions are drawn from the results. The performance with 16 signal paths shows only small variations over different test object orientations. For the test objects used, it is close to the performance in a corresponding 2-D environment with a large number of signal paths, but not necessarily close to the performance in a 3-D environment.

As the number of signal paths is reduced, the primary effect is a strong increase in the spread of the different metrics. Some degradation in average performance is also seen. With only two signal paths the correlation coefficient can have any value, with a significant portion of the values being above 0.7 , even on a test object with good performance in a 3-D environment with many signal paths. Two test objects of the same type of terminal, but with different antenna solutions, are demonstrated to both have very strong performance variations in an environment with limited number of signal paths, but also somewhat different characteristics in terms of median and worst case performance. Which antenna solution is the better depends on the exact number of signal paths, and the choice of performance parameter.

Presented experiments show that characterization in environments with a limited number of signal paths provides a deeper insight into dual antenna performance compared to the traditional characterization assuming a large number of signal paths, and that an MPS is suitable for such characterization.

\section{FUTURE WORK}

Future work includes a study of the same type as the presented, but with the application of realistic channel models to the MPS. Further, models and metrics intended specifically for dual antenna performance in sparse environments should be studied, and specific test methods should be developed that enable performance assessment with maximum reliability at minimal cost.

\section{REFERENCES}

[1] J. Villanen, P. Suvikunnas, C. Icheln, J. Ollikainen, and P. Vainikainen, "Performance analysis and design aspects of mobile-terminal multiantenna configurations," IEEE Trans. Veh. Technol., vol. 57, no. 3, pp. 1664-1674, May 2008.

[2] C. B. Dietrich, Jr., K. Dietze, J. R. Nealy, and W. L. Stutzman, "Spatial, polarization, and pattern diversity for wireless handheld terminals," IEEE Trans. Antennas Propag., vol. 49, no. 9, pp. 1271-1281, Sept. 2001.

[3] T. Taga, "Analysis for mean effective gain of mobile antennas in land mobile radio environments," IEEE Trans. Veh. Technol., vol. 39, no. 2, pp. 117-131, May 1990.

[4] M. J. Gans, "A power-spectral theory of propagation in the mobileradio environment," IEEE Trans. Veh. Technol., vol. VT-21, no. 1, pp. 27-38, Feb. 1972.

[5] P. L. Carro, J. de Mingo, and P. G. Ducar, "Analysis of the antenna stochastic effective gain in mobile environments," presented at the IEEE 69th Veh. Technol. Conf., Barcelona, Spain, Apr. 26-29, 2009.

[6] K. Kalliola, "Experimental Analysis of Multidimensional Radio Channels," Ph.D. dissertation, Helsinki University of Technology, Radio Laboratory Publications, Rep. S 251, Espoo, Finland, Feb. 2002.
[7] A. M. Sayeed and V. Raghavan, "Maximizing MIMO capacity in sparse multipath with reconfigurable antenna arrays," IEEE J. Sel. Topics Signal Process., vol. 1, no. 1, pp. 156-166, June 2007.

[8] J. S. Colburn, Y. Rahmat-Samii, M. A. Jensen, and G. J. Pottie, "Evaluation of personal communications dual-antenna handset diversity performance," IEEE Trans. Veh. Technol., vol. 47, no. 3, pp. 737-746, Aug. 1998.

[9] T. Maeda, S. Sekine, S. Obayashi, and T. Morooka, "Two methods for estimating the diversity characteristics of built-in antennas for mobile communication equipment," in Proc. IEEE AP-S Int. Symp., Jun. 1995, vol. 4, pp. 18-23, pp. 1944-1947.

[10] J. F. Valenzuela-Valdés, A. M. Martínez-González, and D. A. SánchezHernández, "Diversity gain and MIMO capacity for nonisotropic environments using a reverberation chamber," IEEE Antennas Wireless Propag. Lett., vol. 8, pp. 112-115, 2009.

[11] T. Sakata, A. Yamamoto, H. Iwai, K. Ogawa, J. Takada, K. Sakaguchi, and K. Araki, "BER evaluation system for a handset antenna in a multipath environment using a spatial fading emulator," in Proc. ISAP2005, Seoul, Korea, vol. TB1-6, pp. 351-354.

[12] L. Rudant, C. Delaveaud, and M. AbouElAnouar, "Synthesizing realistic environments in an anechoic chamber," in Proc. 3rd Eur. Conf. on Antennas Propag., Berlin, Germany, Mar. 23/27, 2009, pp. 221-225.

[13] P. Hallbjörner, Z. Ying, M. Håkansson, C. Wingqvist, T. Anttila, and J. Welinder, "Multipath simulator for mobile terminal antenna characterization," IET Microw. Antennas Propag., vol. 4, no. 6, pp. 743-750, 2010.

[14] R. Tian, B. K. Lau, and Z. Ying, "Multiplexing efficiency of MIMO antennas," IEEE Antennas Wireless Propag. Lett., vol. 10, pp. 183-186, 2011.

[15] D. G. Brennan, "Linear diversity combining techniques," in Proc. IRE, Jun. 1959, pp. 1075-1102.

[16] S. Saario, D. V. Thiel, J. W. Lu, and S. G. O'Keefe, “An assessment of cable radiation effects on mobile communications antenna measurements," in Proc. IEEE Antennas Propag. Society Int. Symp., Montreal, Canada, Jul. 13/18, 1997, vol. 1, pp. 550-553.

[17] C. Icheln, J. Krogerus, and P. Vainikainen, "Use of balun chokes in small-antenna radiation measurements," IEEE Trans. Instrum. Meas., vol. 53, no. 2, pp. 498-506, Apr. 2004.

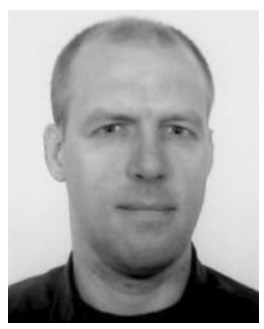

Paul Hallbjörner was born in Uppsala, Sweden, in 1966. He received the B.Sc., M.Sc., and Ph.D. degrees, all in electrical engineering, from Chalmers University of Technology, Göteborg, Sweden, in 1988,1995 , and 2005, respectively.

Since 1989, he has worked in the telecom industry in the areas of pre-production engineering, product development, and research, mainly in the field of antennas and microwave technology. He has worked with mobile terminal antennas for handsets and vehicles, base station antennas, reconfigurable and steerable antennas, wave propagation, passive microwave circuits, electrical material characterization, and millimeter-wave design. He has been employed by Ericsson, Saab, Allgon, and is currently working an Antenna Researcher at SP Technical Research Institute of Sweden, Borås, Sweden, where his main focus is on antenna measurement techniques for mobile phones and short range communication, in addition to antenna development for various applications. He is the author of more than 70 scientific publications and the inventor of 10 patents.

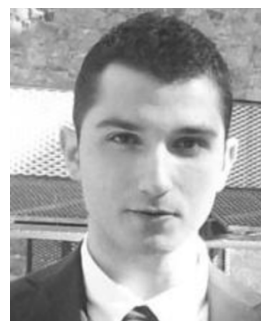

Juan D. Sánchez-Heredia was born in Lorca, Spain. He received the Telecommunication Engineering degree from the Universidad Politécnica de Cartagena, in 2009, which culminated with the Final Degree Award, and the Master degree in information technologies from Universidad de Murcia, in 2010. $\mathrm{He}$ is currently working toward the Ph.D. degree at the Universidad Politécnica de Cartagena, Spain.

In 2007, he worked at General Electric, Cartagena, and was involved in several projects in relation with the network infrastructure. In 2009 he joined the Department of Information Technologies and Communications, as a Ph.D. student. His current research areas cover MIMO communications, multimode-stirred chambers and electromagnetic dosimetry. 


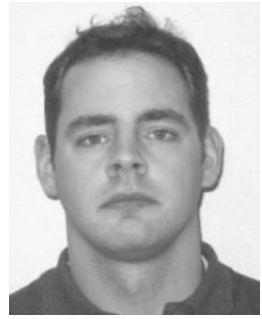

Peter Lindberg (M'08) was born in Uppsala, Sweden, in 1974. He received the M.Sc. and Ph.D. degrees in engineering physics from Uppsala University, Sweden, in 2000 and 2007, respectively.

Previously, he worked as an RF Engineer at Smarteq Wireless, Enebyberg, Sweden, from 2000 and 2002, and as a Research Engineer in the Microwave Technology Group, Uppsala University, from 2002 and 2003. He is the coauthor of more than 30 scientific publications and co-inventor of over 40 patents. Currently, he is with the Advanced Technology Group, Mobile Antenna Systems Division, Laird Technologies, Sweden, working with antenna design, measurement techniques, technology scouting, and customer engineering support.

Dr. Lindberg received the R. W. P. King Best Paper Award from the IEEE Antennas and Propagation Society in 2007.

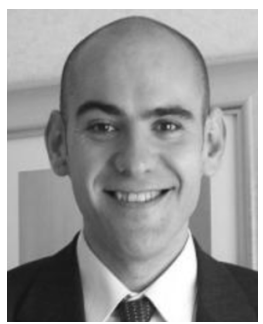

Antonio M. Martínez-González received Dipl.-Ing. degree in telecommunications engineering from the Universidad Politécnica de Valencia, Spain, in 1998 and the Ph.D. degree from Universidad Politécnica de Cartagena, in early 2004.

From 1998 to September 1999, he was employed as a Technical Engineer at the Electromagnetic Compatibility Laboratory, Universidad Politécnica de Valencia, where he developed assessment activities and compliance certifications with European directives related with immunity and emissions to electromagnetic radiation from diverse electrical, electronic and telecommunication equipment. Since September 1999, he is an Associate Professor at Universidad Politécnica de Cartagena. At present, his research interest is focused on electromagnetic dosimetry, radioelectric emissions and mode stirred chambers. In December 2006, he was one of the founders of EMITE Ing, a technological spin-off company founded by telecommunication engineers and doctors of the Microwave, Radiocommunications and Electromagnetism Research Group (GIMRE), Technical University of Cartagena (Spain).

Dr. Martínez-González was awarded with the Spanish National Prize from Foundation Airtel and Colegio Oficial de Ingenieros de Telecomunicación de España for the best final project on Mobile Communications in 1999. In 2006 and 2008, he was a co-recipient (as a co-founder of EMITE) of the i-patentes prize for innovation and technology transfer in the Region of Murcia (Spain).

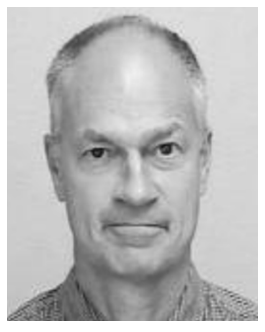

Thomas Bolin was born in Falun, Sweden, in 1954. He received the M.Sc. degree in electrical engineering from Linköping Technical University in Sweden, in 1979

$\mathrm{He}$ is currently working with MIMO antenna design and measurement techniques within Sony Ericsson Mobile Communications, Lund, Sweden. He is one of the pioneers in the mobile handset business having held positions in radio design and management at Ericsson and later Sony Ericsson. Since 1996, he founded and managed an antenna group within his company. From 1979 to 1983, he worked for ITT Standard Radio \& Telefon AB in Vällingby, Sweden, with design of short wave radio kW transmitters. He is the author of a number of scientific papers and holds five patents. 